\title{
Distributed Prescribed Finite Time Consensus Scheme for Economic Dispatch of Smart Grids with the Valve Point Effect
}

\author{
Yingjiang Zhou $\mathbb{D}^{1,2}$ Shigao Zhu, ${ }^{1,2}$ and Qian Chen ${ }^{1,2}$ \\ ${ }^{1}$ College of Automation and College of Artificial Intelligence, Nanjing University of Posts and Telecommunications, \\ Nanjing 210023, China \\ ${ }^{2}$ Jiangsu Engineering Lab for IOT Intelligent Robots (IOTRobot), Nanjing 210023, China \\ Correspondence should be addressed to Yingjiang Zhou; zhouyj@njupt.edu.cn
}

Received 11 July 2020; Revised 10 August 2020; Accepted 19 August 2020; Published 31 August 2020

Academic Editor: Jianquan Lu

Copyright (c) 2020 Yingjiang Zhou et al. This is an open access article distributed under the Creative Commons Attribution License, which permits unrestricted use, distribution, and reproduction in any medium, provided the original work is properly cited.

\begin{abstract}
The distributed prescribed finite time consensus schemes for economic dispatch (ED) of smart grids with and without the valve point effect are researched in this paper. First, the optimization problem is transformed into a consensus of multiagent system problem, where both with and without the valve point effect are considered. Second, for the directed balance network, a prescribed finite time method has been arranged to solve the ED problem with and without the valve point effect. Third, with considering the constraints of generation units, the prescribed finite time result is also achieved. Finally, from the simulations, the efficiency of the proposed algorithms is validated.
\end{abstract}

\section{Introduction}

In the recent years, the research about the ED problem has attracted tremendous attentions, which is particularly important in the smart grid. The aim of the ED problem is to find the optimization of the minimum total generation cost. When there are certain practical constraints, the aim of the ED problem is to find the optimal outputs to minimum total generation cost while meeting the power demand. The traditional ways of solving the ED problem (e.g., genetic algorithm [1,2], particle swarm optimization [3-6], and multiobjective collective decision optimization algorithm [7]) are centralized methods.

Recently, many research results focus on the distributed approach to resolve the ED problem [8-17], where the generation units only get the neighbor's information. The low communication cost, easy implementation and maintenance, and strong robustness against communication uncertainties are the benefits of distributed ED algorithms [8]. Many pioneering work about distributed ED were pointed in [8-10], but these results are not fully distributed. The fully distributed ED algorithms were first proposed in
[11], where the distributed consensus methods in multiagent systems (MASs) have been used. When there exist unknown communication uncertainties, the ED problem is developed by using the adaptive consensus-based robust strategy [12]. For sparse communication networks and time delay, a distributed scheme is provided based on consensus strategy [13]. In order to reduce the amount of communication of the smart grids, the event-triggered control solution was devised to achieve the distributed reactive power sharing control [14-16]. In the smart grids, the second-order consensus methods have been used to solve the ED problem [17]. Considering the complex networks with the reaction diffusion terms and the probabilistic Boolean networks, the synchronization and stabilization methods were investigated in [18-20], and we will focus on the ED algorithms in these kinds of networks.

The rate of convergence is a key factor of solving the ED problem [21-25], and fast convergence rate and strong robustness are the advantages of the finite time method $[26,27]$. Considering the ED problem of generation, the distributed finite-step iterative strategy is arranged [21]. If the topology is jointly connected, distributed finite time ED 
in smart grid is derived [22]. In [15, 22], the homogeneous method is applied to solve the finite time ED problem, but it is hard to estimate the convergence time. A novel fully distributed finite time algorithm is devised to address the ED problem in the smart grids [23]. The convergence time in the abovementioned algorithms is connected with the initial condition, if the initial condition is large, the convergence time will be very long. The prescribed finite time method was proposed for the first time in [24], where the convergence time can be set by the designer. Very recently, a distributed prescribed finite time method for the ED problem was proposed [25].

Another important topic is about the valve point effect [28-41]. Many meaningful works are focused on the valve point effect, such as evolutionary programming [28, 29], genetic algorithm [30-33], and particle swarm optimisation $[34,35]$. The complicated, nonlinear, and nonconvex ED problem with the valve point effect has been settled by using the Maclaurin series-based Lagrangian algorithm [36]. In [37], the valve point loading effect and transmission losses are considered, where the ED problem has been resolved with the gradient and Newton methods. But, most algorithms are centralized, and relatively speaking, distributed methods are more consistent with the actual requirements. In [38], the ED problem with the valve point effect is resolved with a distributed pattern search scheme.

The contributions of this paper can be listed in the following three aspects. (1) For the directed balance network, a prescribed finite time approach has been pointed out to solve the ED problem. (2) The prescribed finite time method was also derived for solving the ED problem with considering the valve point effect in this paper. (3) With considering the constraints of generation units, the prescribed finite time result is still correct.

The remainder of this article is organized as follows. The graph theory and problem statement are introduced in Section 2. Section 3 proposes distributed prescribed finite time consensus algorithms of solving the optimization without and with the valve point effect and the generation constraints of generation units. Three simulation examples are proposed to validate our results in Section 4. In Section 5, the conclusion has been presented.

\section{Graph Theory and Problem Statement}

In this section, the graph theory and the problem statement are introduced.

2.1. Graph Theory. We define $G=(V, E, A)$ as a weighted directed graph, where $V=\left\{v_{1}, \ldots, v_{n}\right\}$ represents the set of nodes, $E \subseteq V \times V$ represents the set of edges, $\left(v_{i}, v_{j}\right) \in E$ represents that nodes $i$ can receive information from node $j$, and $A=\left[a_{i j}\right] \in R^{n \times n}$ is the weighted adjacency matrix. If and only if there is a directed edge $\left(v_{i}, v_{j}\right)$ in $G$, then the weight of the edge $a_{i j}>0$; otherwise, $a_{i j}=0,(i \neq j, i, j=1, \ldots, n)$. Assume that $a_{i i}=0$ for all $i \in\{1, \ldots, n\}$. The Laplacian matrix of a directed graph $G$ is $L=\left[l_{i j}\right] \in R^{n \times n}$, where $l_{i i}=$ $\sum_{j=1}^{n} a_{i j}$ and $l_{i j}=-a_{i j}, i \neq j, i, j=1, \ldots, n$.
The direct path from the node $i_{1}$ to node $i_{m}$ is $\left(i_{1}, i_{2}\right),\left(i_{2}, i_{3}\right), \ldots,\left(i_{m-1}, i_{m}\right)$. If one node in a directed graph at least has a directed path to another node, the graph contains a directed spanning tree. If each node has a directed path to all other nodes in a directed graph, the directed graph is said to be strongly connected. If all the nodes satisfy $d_{\text {in }}(i)=\sum_{j=1}^{N} a_{i j}=d_{\text {out }}(i)=\sum_{j=1}^{N} a_{j i}, i, j=1, \ldots, n$ and the in-degree is equal to the out-degree, then the direct graph is said to be a balance graph.

Define an undirected mirror graph $\widehat{G}=\{V, \widehat{E}, \widehat{A}\}$, which has the same nodes $V$ as $G$. The set of edges is $\widehat{E} \subseteq V \times V$, and the weighted symmetric adjacency matrix is $\widehat{A}=\left[\widehat{a}_{i j}\right]$, where

$$
\widehat{a}_{i j}=\widehat{a}_{j i}=\frac{a_{i j}+a_{j i}}{2} \geq 0 .
$$

2.2. Problem Statement. Suppose the MASs consist of $n$ agents. Also, we can describe the dynamics of the agent as follows:

$$
\dot{x}_{i}=u_{i}
$$

where $u_{i}$ is the control inputs, $i=1, \ldots, n$.

Definition 1. The MASs (2) is said to reach consensus in prescribed finite time, if for any preselected time $T>0$ such that $\lim _{t \longrightarrow T} x_{i}=x_{j}$ and for all $t \geq T, \quad x_{i}=x_{j}$, $i, j \in\{1, \ldots, n\}$.

Lemma 1 (see [10]). For the irreducible Laplacian matrix $L$, the algebraic connectivity $a(L)>0$, where $a(L)=\min _{x^{T} \xi=0, x \neq 0}$ $x^{T} \widehat{L} x / x T \Xi x$, with $\widehat{L}=\Xi L+L^{T} \Xi / 2, \quad \xi=\left(\xi_{1}, \xi_{2}, \ldots, \xi_{n}\right)>0$, $\Xi=\operatorname{diag}\left(\xi_{1}, \ldots, \xi_{n}\right), \sum_{i=1}^{n} \xi_{i}=1$, and $\xi^{T} L=0$.

In this paper, the ED problem with and without the valve point effect is considered. Suppose there are $n$ generating units. The cost function of each generator is as follows:

$$
C_{i}(P)_{i}=a_{i} P_{i}^{2}+b_{i} P_{i}+c_{i}+d_{i}\left|\sin \left(e_{i}\left(P_{i}^{\min }-P_{i}\right)\right)\right|,
$$

where $C_{i}\left(P_{i}\right)$ is the cost of the $i$-th generator, $P_{i}$ is the real power generation of the $i$-th unit, $P_{i}^{\min }$ is the lower bound of the generation capacity, and $a_{i}, b_{i}, c_{i}, d_{i}$, and $e_{i}$ are the positive cost function coefficients. $d_{i}\left|\sin \left(e_{i}\left(P_{i}^{\min }-P_{i}\right)\right)\right|$ is the valve point effect. If $d_{i}=0$, it means that the valve point effect is not exist.

Our research objective is to minimize the total cost of $n$ power generation systems in the case of power demand and supply balance.

The optimization problem can be summarized as follows:

$$
\min \sum_{i=1}^{n} C_{i}\left(P_{i}\right)
$$

Subjecting to the power balance constraint,

$$
\sum_{i=1}^{n} P_{i}=P_{D}
$$

where $P_{D}$ is the total load of the power system. 


\section{Main Theoretical Results}

3.1. Optimization by Consensus without the Valve Point Effect. In this section, the distributed control method is devised to solve the optimization problem without the valve point effect.

Definition 2. The incremental cost of each generator $i$ without valve point effect is defined as $\mathrm{IC}_{i}=\partial C_{i}\left(P_{i}\right) / \partial P_{i}=r_{i} P_{i}+b_{i}, i=1,2, \ldots, n$, where $r_{i}=2 a_{i}$.

The Lagrange multiplier algorithm is applied to solve the optimization problem.

$$
\begin{aligned}
L\left(P_{i}, \lambda\right) & =\sum_{i=1}^{n} C_{i}\left(P_{i}\right)+\lambda\left(P_{D}-\sum_{i=1}^{n} P_{i}\right) \\
& =\sum_{i=1}^{n}\left(a_{i} P_{i}^{2}+b_{i} P_{i}+c_{i}\right)+\lambda\left(P_{D}-\sum_{i=1}^{n} P_{i}\right),
\end{aligned}
$$

where $\lambda$ is the Lagrange multiplier.

The minimum value of (6) can be obtained by differentiating the abovementioned equation.

$$
\frac{\partial L}{\partial P_{i}}=\frac{\partial C_{i}\left(P_{i}\right)}{\partial P_{i}}-\lambda=r_{i} P_{i}+b_{i}-\lambda=0 .
$$

So, $\sum_{i=1}^{n} P_{i}=P_{D}$. We have

$$
\lambda=r_{i} P_{i}+b_{i} \text {. }
$$

We define $y_{i}(t)=\lambda_{i}=r_{i} P_{i}+b_{i}$. Then, a distributed prescribed finite time protocol is designed for the optimization problem.

$$
\dot{y}_{i}(t)=\operatorname{cr}_{i} k(t) \sum_{j=1}^{n} \widehat{a}_{i j}\left(y_{j}(t)-y_{i}(t)\right)=-\operatorname{cr}_{i} k(t) \sum_{j=1}^{n} \widehat{L}_{i j} y_{j}(t) .
$$

With initial conditions $y_{i}(0)=r_{i} P_{i}(0)+b_{i}$, the following equation is satisfied:

$$
\sum_{i=1}^{n} P_{i}(0)=P_{D}
$$

$k(t)=k_{1}+k_{2} / T-t \operatorname{sign}[1+\operatorname{sign}(T-t)], k_{1}>0, k_{2}>0$, and $c \geq 1 / a\left(\Xi^{-1} \widehat{L}\right)>0$ are constants. $T>0$ is a prespecifiable convergence time.

From [10], condition (10) is satisfied.

Theorem 1. If the topological graph is a strongly connected direct balance graph, the distributed prescribed finite time protocol (9) is designed to solve the prescribed finite time optimization problem without the valve point effect (4) via the initial conditions in (10).

Proof. First, we will prove the balance between demand and supply of powers.

Let $\xi_{m}=1 / r r_{m}$, and $y^{*}(t)=\sum_{m=1}^{n} \xi_{m} y_{m}(t)=(1 / r)$ $\sum_{m=1}^{n}\left(b_{m} / r_{m}+P_{m}(t)\right)$ be the weighted average value of all the incremental costs, where $r=\sum_{m=1}^{n} 1 / r_{m}, m=1,2, \ldots, n$. We know $\widehat{L}$ is zero row-sum and symmetric, so

$$
\dot{y}^{*}(t)=-\frac{\operatorname{ck}(t)}{r} \sum_{m=1}^{n} \sum_{j=1}^{n} \widehat{L}_{m j} y_{j}(t)=0=\frac{1}{r} \sum_{m=1}^{n} \dot{P}_{m}(t) .
$$

This means

$$
\sum_{m=1}^{n} P_{m}(t)=\sum_{m=1}^{n} P_{m}(0)=P_{D}
$$

From (12), we know the total output power of all the generators is a constant value, which means the balance between the demand and supply of powers is always true.

Then, we will prove the prescribed finite time consensus for the incremental cost of each generator.

We define $\delta_{i}=y_{i}-y^{*}$ as the error states between the average value and the $i$-th generator, $\delta=\left(\delta_{1}, \ldots, \delta_{n}\right)^{T}$. It is easy to see that $\sum_{k=1}^{n} \xi_{k}=1$ and $\sum_{k=1}^{n} \xi_{k} \delta_{k}=0$. So, we can get the error system:

$$
\dot{\delta}_{i}(t)=\operatorname{cr}_{i} k(t) \sum_{j=1}^{n} \widehat{a}_{i j}\left(\delta_{j}(t)-\delta_{i}(t)\right)=-\operatorname{cr}_{i} k(t) \sum_{j=1}^{n} \widehat{L}_{i j} \delta_{j}(t) .
$$

We choose the following Lyapunov function:

$$
V(t)=\sum_{i=1}^{n} \frac{\delta_{i}^{T} \delta_{i}}{r_{i}}
$$

By using Lemma 1, the derivative of $V(t)$ can be described as

$$
\begin{aligned}
\dot{V}(t) & =2 \sum_{i=1}^{n} \frac{\delta_{i}^{T} \dot{\delta}_{i}}{r_{i}}=-2 \operatorname{ck}(t) \sum_{i=1}^{n} \sum_{j=1}^{n} \delta_{i}^{T} \widehat{L}_{i j} \delta_{j}=-2 \operatorname{ck}(t) \delta^{T} \widehat{L} \delta \\
& \leq-\operatorname{ck}(t) a\left(\Xi^{-1} \widehat{L}\right) \delta^{T} \Xi \delta \leq-\operatorname{ck}(t) a\left(\Xi^{-1} \widehat{L}\right) V(t) .
\end{aligned}
$$

As $k(t)=k_{1}+k_{2} / T-t \operatorname{sign}[1+\operatorname{sign}(T-t)]$, we will prove the result in two intervals $[0, T)$ and $[T, \infty)$.

For $t \in[0, T), k(t)=k_{1}+k_{2} / T-t$. We have

$$
\begin{aligned}
\dot{V}(t) \leq & -c\left(k_{1}+\frac{k_{2}}{T-t}\right) a\left(\Xi^{-1} \widehat{L}\right) V=-\operatorname{ck}_{1} a\left(\Xi^{-1} \widehat{L}\right) V \\
& -c \frac{k_{2}}{T-t} a\left(\Xi^{-1} \widehat{L}\right) V .
\end{aligned}
$$

We define $z=(T-t)^{-k_{2} / 2}, t \in[0, T)$. Then, $\dot{z}=k_{2} / 2$ $(T-t)^{-\left(k_{2} / 2\right)-1}, \quad \dot{z} / z=k_{2} / 2(T-t)$. As $c \geq 1 / a\left(\Xi^{-1} \widehat{L}\right)>0$, we have

$$
\dot{V}(t) \leq-k_{1} V(t)-\frac{k_{2}}{T-t} V(t)=-k_{1} V(t)-2 \frac{\dot{z}}{z} V(t) .
$$

Then,

$$
z^{2} \dot{V}(t) \leq-k_{1} z^{2} V(t)-2 z \dot{z} V(t)
$$

So, 


$$
\frac{\mathrm{d}\left(z^{2} V(t)\right)}{\mathrm{d} t}=z^{2} \dot{V}(t)+2 z \dot{z} V(t) \leq-k_{1} z^{2} V(t)
$$

Then,

$$
\begin{aligned}
z^{2} V(t) & \leq \exp ^{-k_{1} t} z(0)^{2} V(0), \\
V(t) & \leq z^{-2} \exp ^{-k_{1} t} z(0)^{2} V(0)=\left(\frac{T-t}{T}\right)^{2} \exp ^{-k_{1} t} V(0) .
\end{aligned}
$$

From (21), we know $\lim _{t \rightarrow T^{-}} V(t)=0$.

For $t \in[T, \infty), k(t)=k_{1}$. We have

$$
\dot{V}(t) \leq-\mathrm{ck}_{1} a\left(\Xi^{-1} \widehat{L}\right) V<0 .
$$

It means $V$ will not rise anymore. So, $\forall t \geq T$, $V(t)=V(T) \equiv 0$.

The prescribed finite time optimization problem without the valve point effect has been proved. The proof is completed.

Remark 1: the prescribed finite time fully distributed method is designed for solving the ED problem in Theorem 1 . The result here is a fully distributed result, and it is different from the centralized algorithms.

Remark 2: note that the graph in Theorem 1 is a direct strongly connected balance graph, and it is an improvement over an undirected graph. The result here can also be used for any undirected connected graph. In the future work, we will focus on other direct graphs.

Remark 3: the prescribed finite time result has two advantages compared with the finite time result. Firstly, the convergence time can be preassigned as needed by the designer, as we know that the convergence time of the finite time results is connected with the initial condition. Secondly, the controller in the prescribed finite time result is continuous, while the controller in the finite time result is discontinuous. The continuous controller can make the system state change smoothly.

3.2. Valve Point Effect. In this section, the valve point effect is introduced. In the interval $[0, \pi]$, the piecewise linearization is employed. The effect of the valve point effect on the cost function is the type of the sine-wave function, as in Figure 1(a). The active real power generated can obtained from the derivative of the sine-wave function, as in Figure 1(b). As the function in Figure 1(b) is periodic, the piecewise linearization is introduced to approximate the sine-wave function, as in Figure 1(c) [42].

Taking the derivative of formula (3),

$$
\frac{\mathrm{d} C_{i}\left(P_{i}\right)}{\mathrm{d}\left(P_{i}\right)}=2 a_{i} P_{i}+b_{i}+d_{i} e_{i} \cos \left(\bmod \left(e_{i}\left(P_{i}^{\min }-P_{i}\right), \pi\right)\right),
$$

where mod means the MOD function.
The piecewise linearization with different slopes is introduced:

$$
d_{i} e_{i} \cos \left(\bmod \left(e_{i}\left(P_{i}^{\min }-P_{i}\right), \pi\right)\right)=g_{i}\left(P_{i}-q_{i}\left(\frac{\pi}{e_{i}}\right)\right)+k_{i},
$$

where $g_{i}$ and $k_{i}$ are constants and $q_{i}=\left[\left(P_{i}-P_{i}^{\min }\right) /\left(\pi / e_{i}\right)\right]$ is an integer number of intervals. From (23) and (24),

$$
\begin{aligned}
\frac{\mathrm{d} C_{i}\left(P_{i}\right)}{\mathrm{d}\left(P_{i}\right)} & =2 a_{i} P_{i}+b_{i}+g_{i}\left(P_{i}-q_{i}\left(\frac{\pi}{e_{i}}\right)\right)+k_{i} \\
& =\left(2 a_{i}+g_{i}\right) P_{i}-g_{i} q_{i}\left(\frac{\pi}{e_{i}}\right)+b_{i}+k_{i} .
\end{aligned}
$$

3.3. Optimization by Consensus with the Valve Point Effect. Next, the distributed control approach is devised of solving the optimization problem with the valve point effect.

Definition 3. We define the incremental cost of each generator $i$ as $I C_{i}=\partial C_{i}\left(P_{i}\right) / \partial P_{i}=\bar{r}_{i} P_{i}+\eta_{i}$, where $\bar{r}_{i}=2 a_{i}+g_{i}$ and $\eta_{i}=-g_{i} q_{i}\left(\pi / e_{i}\right)+b_{i}+k_{i}, i=1,2, \ldots, n$.

The Lagrange multiplier algorithm is used to solve the optimization problem with the valve point effect.

$$
\begin{aligned}
L\left(P_{i}, \lambda\right)= & \sum_{i=1}^{n} C_{i}\left(P_{i}\right)+\lambda\left(P_{D}-\sum_{i=1}^{n} P_{i}\right) \\
= & \sum_{i=1}^{n} a_{i} P_{i}^{2}+b_{i} P_{i}+c_{i}+d_{i}\left|\sin \left(e_{i}\left(P_{i}^{\min }-P_{i}\right)\right)\right| \\
& +\lambda\left(P_{D}-\sum_{i=1}^{n} P_{i}\right)
\end{aligned}
$$

The minimum value of (26) can be obtained by differentiating equation (26).

$$
\frac{\partial L}{\partial P_{i}}=\frac{\partial C_{i}\left(P_{i}\right)}{\partial P_{i}}-\lambda=\bar{r}_{i} P_{i}+\eta_{i}-\lambda=0 .
$$

So, $\sum_{i=1}^{n} P_{i}=P_{D}$. We have

$$
\lambda=\bar{r}_{i} P_{i}+\eta_{i} \text {. }
$$

We define $x_{i}(t)=\lambda_{i}=\bar{r}_{i} P_{i}+\eta_{i}, i=1,2, \ldots, n$. Then, a distributed prescribed finite time protocol is designed for the optimization problem.

$$
\dot{x}_{i}(t)=\mathrm{cr}_{i} k(t) \sum_{j=1}^{n} \widehat{a}_{i j}\left(x_{j}(t)-x_{i}(t)\right)=-\mathrm{cr}_{i} k(t) \sum_{j=1}^{n} \widehat{L}_{i j} x_{j}(t) .
$$

With initial conditions $x_{i}(0)=\bar{r}_{i} P_{i}(0)+\eta_{i}$, the following equation is satisfied:

$$
\sum_{i=1}^{n} P_{i}(0)=P_{D} .
$$




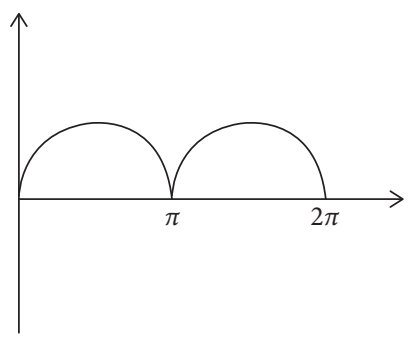

(a)

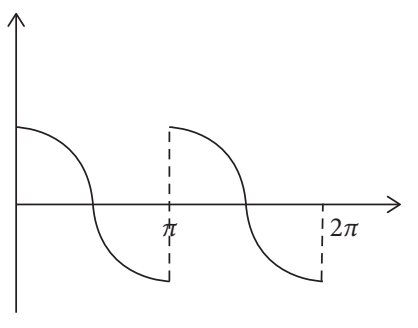

(b)

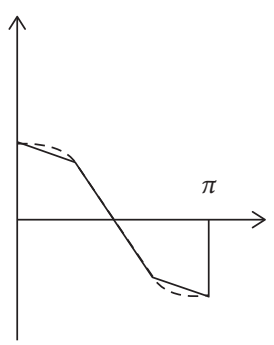

(c)

Figure 1: Piecewise linearization of the sine-wave part.

$k(t)=k_{1}+k_{2} / T-t \operatorname{sign}[1+\operatorname{sign}(T-t)], c \geq 1 / a\left(\Xi^{-1} \widehat{L}\right)$ $>0, k_{1}>0, k_{2}>0$ are constants. $T>0$ is a prespecifiable convergence time.

Theorem 2. If the topological graph is a strongly connected direct balance graph, the distributed prescribed finite time protocol (29) is designed to solve the prescribed finite time optimization problem with the valve point effect (4) via the initial conditions in (30).

The proof here is similar to the proof in Theorem 1. We omitted the proof here.

3.4. Optimization by Consensus with the Power Generation Constraints. Next, considering the power generation constraints of the generation-demand constraint, we need to further revise the distributed algorithms (9)-(10) to solve the ED problem.

The following three steps are derived to solve the constraints problem:

Step 1: by using the algorithm (9)-(10) in Theorem 1, we can get the optimal incremental cost $\lambda^{*}$ and the optimal power generation value $P_{i}^{*}$.

Step 2: from Step 1, we get $P_{i}^{*}$. We check to see whether $P^{*}$ is in the interval $\left[P_{i, \text { min }}, P_{i, \max }\right]$. If $P_{i}^{*}>P_{i, \max }$, let $P_{i}^{*}=P_{i, \max }$. If $P_{i}^{*}<P_{i, \min }$, let $P_{i}^{*}=P_{i, \text { min }}$.

We define $\Omega_{p}$ as the generation units whose optimal values of power generation are $P_{i}^{*}=P_{i, \max }$ or $P_{i}^{*}=P_{i, \min }$. Two auxiliary variables $\hat{x}_{i}, \hat{y}_{i}$ are introduced, and the initialize condition are

$$
\begin{aligned}
& \widehat{x}_{i}= \begin{cases}\frac{\lambda^{*}-b_{i}}{2 a_{i}}-P_{i}^{*}, & i \in \Omega_{p}, \\
0, & i \notin \Omega_{p},\end{cases} \\
& \widehat{y}_{i}= \begin{cases}\frac{1}{2 a_{i}}, & i \notin \Omega_{p}, \\
0, & i \in \Omega_{p} .\end{cases}
\end{aligned}
$$

The distributed average algorithms are introduced:

$$
\begin{aligned}
& \dot{\hat{x}}_{i}(t)=\operatorname{cr}_{i} k(t) \sum_{j=1}^{n} \widehat{a}_{i j}\left(\hat{x}_{j}(t)-\widehat{x}_{i}(t)\right), \\
& \dot{\hat{y}}_{i}(t)=\operatorname{cr}_{i} k(t) \sum_{i=1}^{n} \hat{a}_{i j}\left(\hat{y}_{j}(t)-\hat{y}_{i}(t)\right) .
\end{aligned}
$$

Corollary 1. If the topological graph is a strongly connected direct balance graph, the distributed prescribed finite time algorithms (32) and (33) can solve the optimization problem with the power generation constraints in the preselected finite time $T_{1}$, i.e.,

$$
\begin{aligned}
& \lim _{t \longrightarrow T_{1}} \hat{x}^{*}(t)=\sum_{i=1}^{n} \xi_{i} \hat{x}_{i}(0)=\sum_{i \in \Omega_{p}} \xi_{i}\left(\frac{\lambda^{*}-b_{i}}{2 a_{i}}-P_{i}^{*}\right), \\
& \lim _{t \longrightarrow T_{1}} \hat{y}^{*}(t)=\sum_{i=1}^{n} \xi_{i} \hat{y}_{i}(0)=\sum_{i \in \Omega_{p}} \frac{\xi_{i}}{2 a_{i}} .
\end{aligned}
$$

Proof. When we set $a_{i}=0.5$, it is easy to get the results (34) and (35), and the proof is similar to the proof in Theorem 1.

From (34) and (35), each generation unit obtains the average values of $\hat{x}_{i}^{*}$ and $\hat{y}_{i}^{*}$. We can get the new incremental cost $\lambda^{* *}$ as follows:

$$
\lambda^{* *}=\lambda^{*}+\frac{\widehat{x}_{i}^{*}}{\widehat{y}_{i}^{*}} .
$$

The new optimal value $P_{i}^{* *}$ can be obtained by

$$
P^{* *}=\left\{\begin{array}{ll}
\frac{\lambda^{* *}-b_{i}}{2 a_{i}} & i \notin \Omega_{p} \\
P_{i, \text { min }} \text { or } P_{i, \max } & i \in \Omega_{p}
\end{array} .\right.
$$

Step 3: check to see weather $P^{* *}$ is in the interval $\left[P_{i, \text { min }}, P_{i, \text { max }}\right]$. If $P^{* *}$ is not in the interval, set $\lambda^{*}=\lambda^{* *}$ and repeat Step 2. Otherwise, $P^{* *}$ is the final value. 


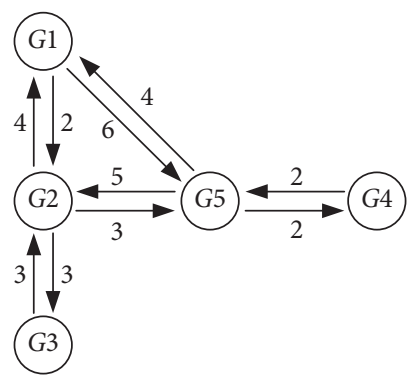

Figure 2: The topology graph of the generator model.

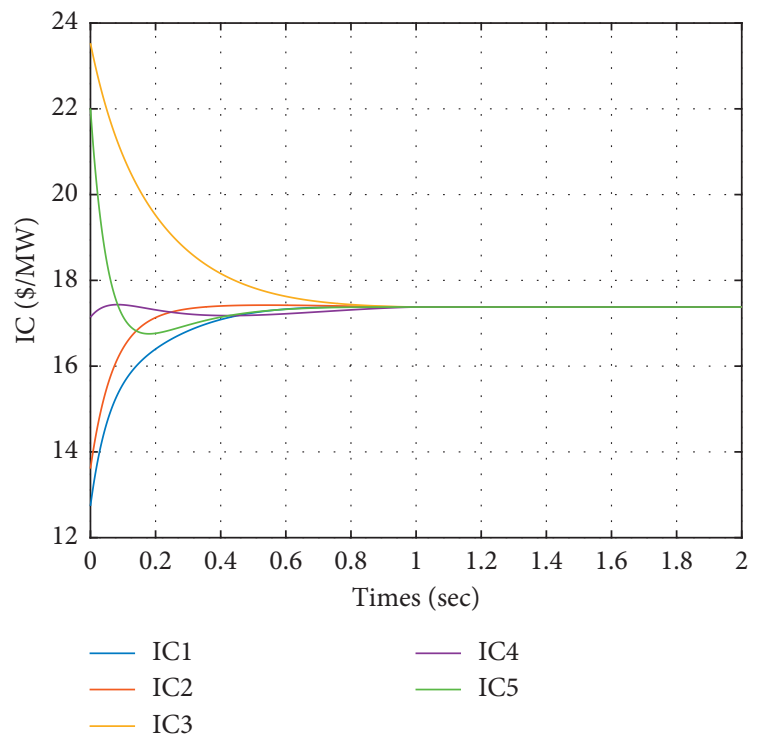

Figure 3: The incremental cost of generator $\lambda_{i}$.

\section{Numerical Examples}

In this section, in order to verify the effectiveness of prescribed finite time algorithm for the ED problem of smart grids with the valve point effect, three numerical examples are listed, prescribed finite time optimization by consensus, prescribed finite time optimization by consensus with power generation constraints of generation units, and prescribed finite time optimization by consensus with the valve point effect. In this paper, a simulation model with 5 generators is selected, and the communication topology of the generator model can be seen in Figure 2.

The topology is balance directed, the adjacent matrix of the graph can be written as $A=\left[\begin{array}{lllll}0 & 2 & 0 & 0 & 6 \\ 4 & 0 & 3 & 0 & 3 \\ 0 & 3 & 0 & 0 & 0 \\ 0 & 0 & 0 & 0 & 2 \\ 4 & 5 & 0 & 2 & 0\end{array}\right]$, and the Laplacian matrix of the MASs is $L=\left[\begin{array}{ccccc}8 & -2 & 0 & 0 & -6 \\ -4 & 10 & -3 & 0 & -3 \\ 0 & -3 & 3 & 0 & 0 \\ 0 & 0 & 0 & 2 & -2 \\ -4 & -5 & 0 & -2 & 11\end{array}\right]$. The mirror diagram can be further obtained as

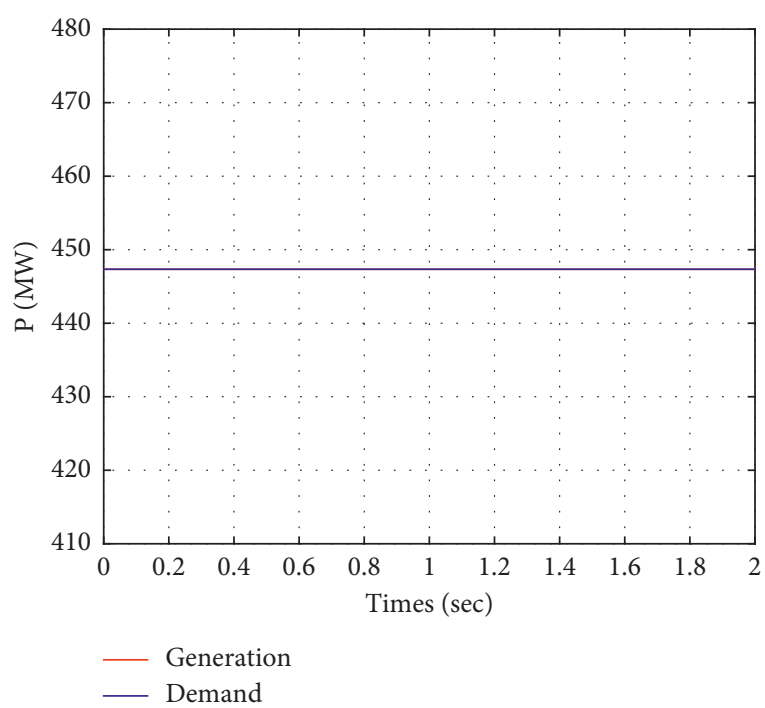

FIgURE 4: Total power demand of the generator.

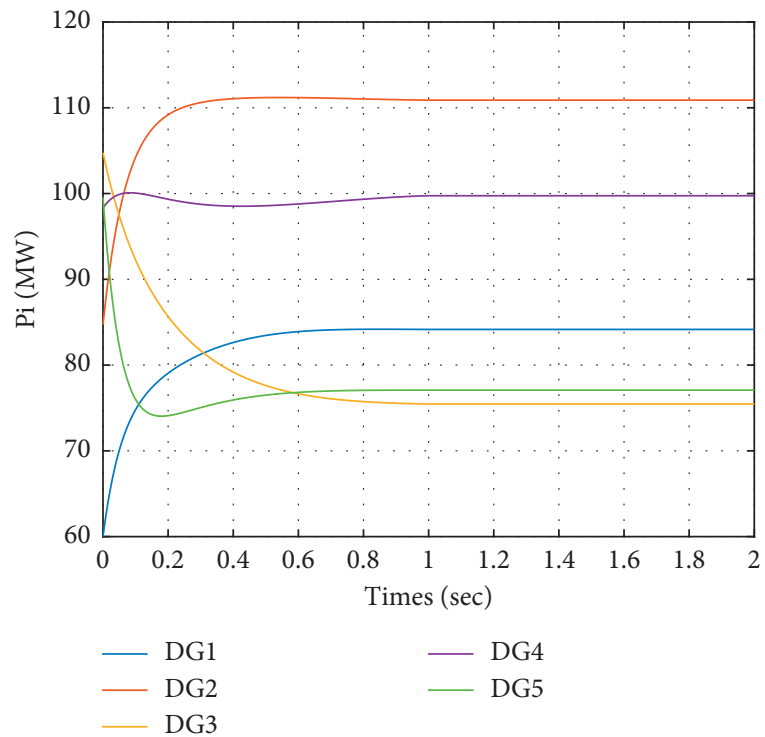

Figure 5: The output power of generator $P_{i}$.

$\widehat{A}=\left[\begin{array}{lllll}0 & 3 & 0 & 0 & 5 \\ 3 & 0 & 3 & 0 & 4 \\ 0 & 3 & 0 & 0 & 0 \\ 0 & 0 & 0 & 0 & 2 \\ 5 & 4 & 0 & 2 & 0\end{array}\right]$, and the Laplacian matrix of mirror diagram is $\widehat{L}=\left[\begin{array}{ccccc}8 & -3 & 0 & 0 & -5 \\ -3 & 10 & -3 & 0 & -4 \\ 0 & -3 & 3 & 0 & 0 \\ 0 & 0 & 0 & 2 & -2 \\ -5 & -4 & 0 & -2 & 11\end{array}\right]$.

For $k(t)=k_{1}+k_{2} / T-t \operatorname{sign}[1+\operatorname{sign}(T-t)], \quad$ select $k_{1}=1, k_{2}=2, c=2$ in equation (9). Set $a_{1}=0.096$, $a_{2}=0.072, a_{3}=0.105, a_{4}=0.082, a_{5}=0.103, b_{1}=1.22$, $b_{2}=1.41, b_{3}=1.53, b_{4}=1.02, b_{5}=1.50, c_{1}=51, c_{2}=31$, $c_{3}=78, c_{4}=42$, and $c_{5}=81$. The original values of $\lambda_{i}(0)$ are 


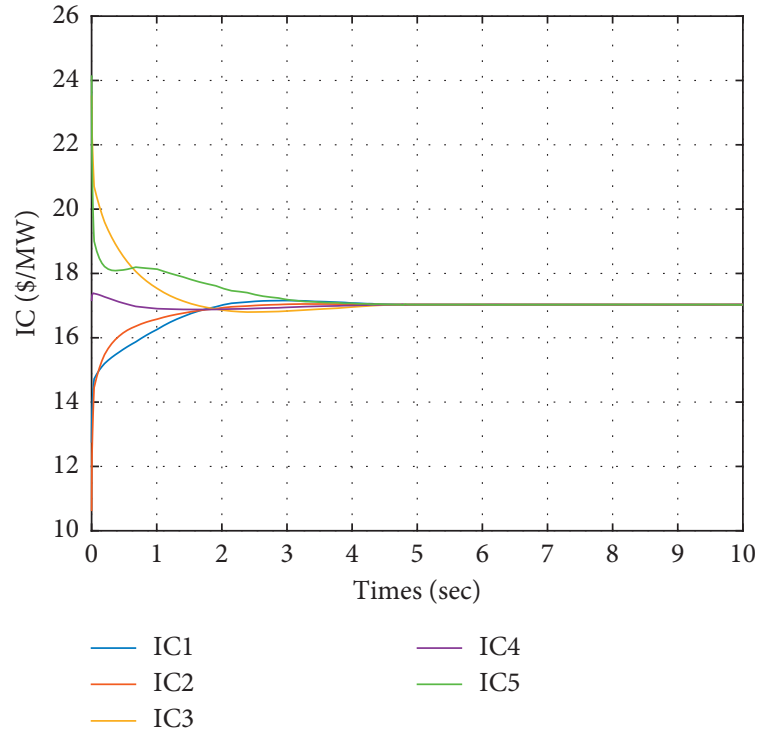

Figure 6: The incremental cost of generator $\lambda_{i}$ with power generation constraints.

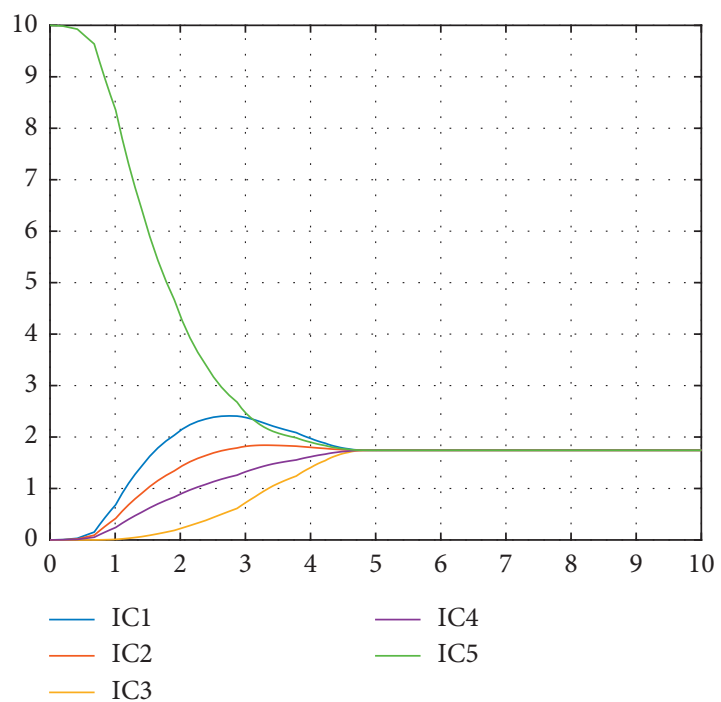

FIgURe 7: The incremental cost of generator $x_{i}$ with power generation constraints.

$\lambda_{1}(0)=12.74, \lambda_{2}(0)=13.61, \lambda_{3}(0)=23.53, \lambda_{4}(0)=17.14$, and $\lambda_{5}(0)=22.01$.

4.1. Prescribed Finite Time Optimization by Consensus. In this section, distributed ED algorithm (9) in a directed topology is used to solve the optimization problem without considering power generation constraints.

The convergence time can be set as $1 \mathrm{~s}$. Figure 3 shows the incremental cost of the generator $\lambda_{i}$, and the stable consensus $\lambda^{*}=17.38$ after $1 \mathrm{~s}$. We can see that the generator power demand is about 447.34 MW from Figure 4, which satisfies the balance condition of power demand. It can be seen from Figure 5 that the optimal values of the generator

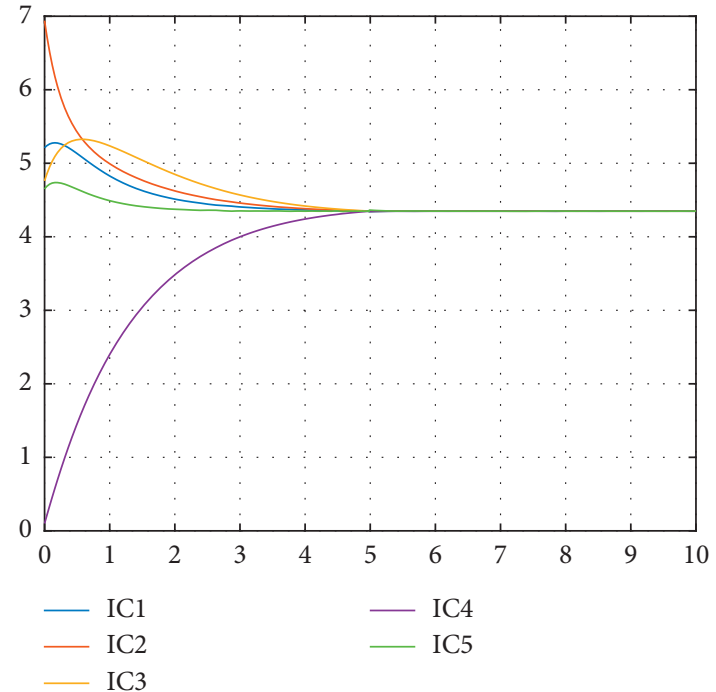

FIgURE 8: The incremental cost of generator $y_{i}$ with power generation constraints.

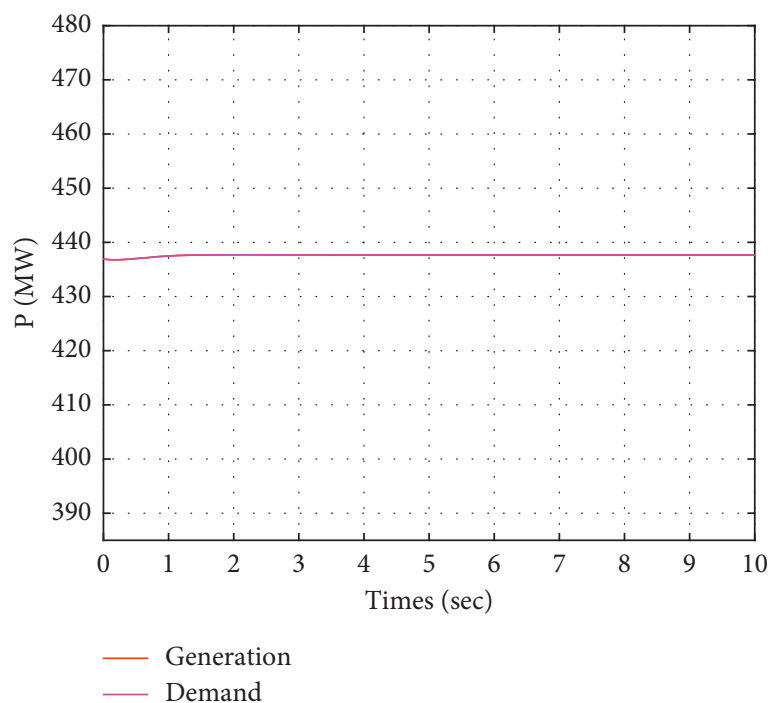

FIgURE 9: Total power demand of generator with power generation constraints.

power output are $P_{1}=84.16 \mathrm{MW}, P_{2}=110.89 \mathrm{MW}$, $P_{3}=75.47 \mathrm{MW}, P_{4}=99.74 \mathrm{MW}$, and $P_{5}=77.08 \mathrm{MW}$.

4.2. Prescribed Finite Time Optimization by Consensus with Power Generation Constraints. Power generation constraints of generator units are taken into account in this simulation. The maximum values of each generator constraints are $P_{1 H}=200, P_{2 H}=190, P_{3 H}=180, P_{4 H}=120$, and $P_{5 H}=180$. The minimum power of each generators are $P_{1 L}=60, P_{2 L}=40, P_{3 L}=50, P_{4 L}=30$, and $P_{5 l}=20$. The initial condition of the two auxiliary variables are $\hat{x}_{1}(0)=0$, $\widehat{x}_{2}(0)=0, \hat{x}_{3}(0)=0, \widehat{x}_{4}(0)=0, \widehat{x}_{5}(0)=10, \hat{y}_{1}(0)=5.21$, $\hat{y}_{2}(0)=6.94, \hat{y}_{3}(0)=4.76, \widehat{y}_{4}(0)=0.10$, and $\hat{y}_{5}(0)=4.65$. The convergence time is selected as $5 \mathrm{~s}$. The incremental cost of the generator is $\lambda^{*}=17.03$, as in Figure 6. According to 


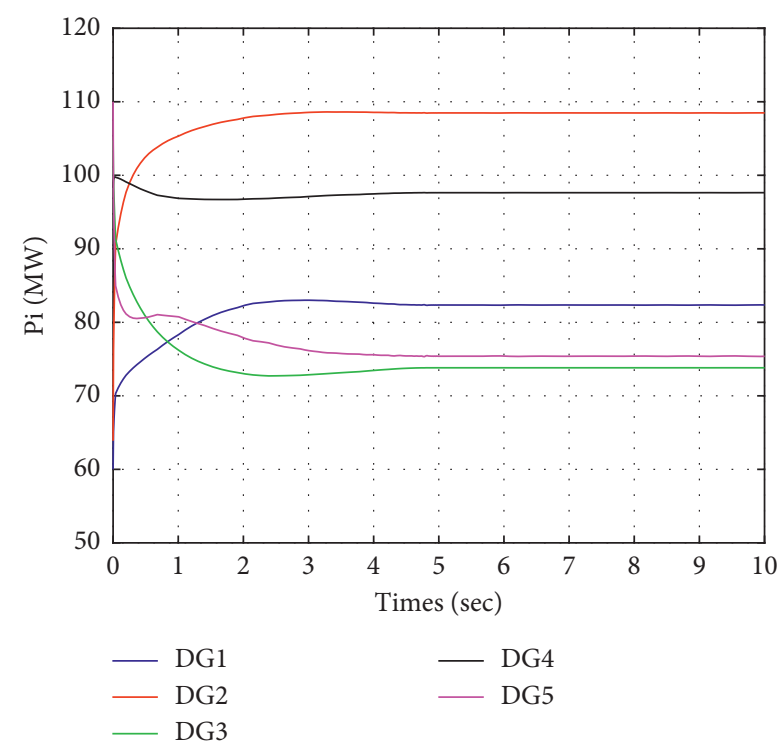

Figure 10: The output power of generator $P_{i}$ with power generation constraints.

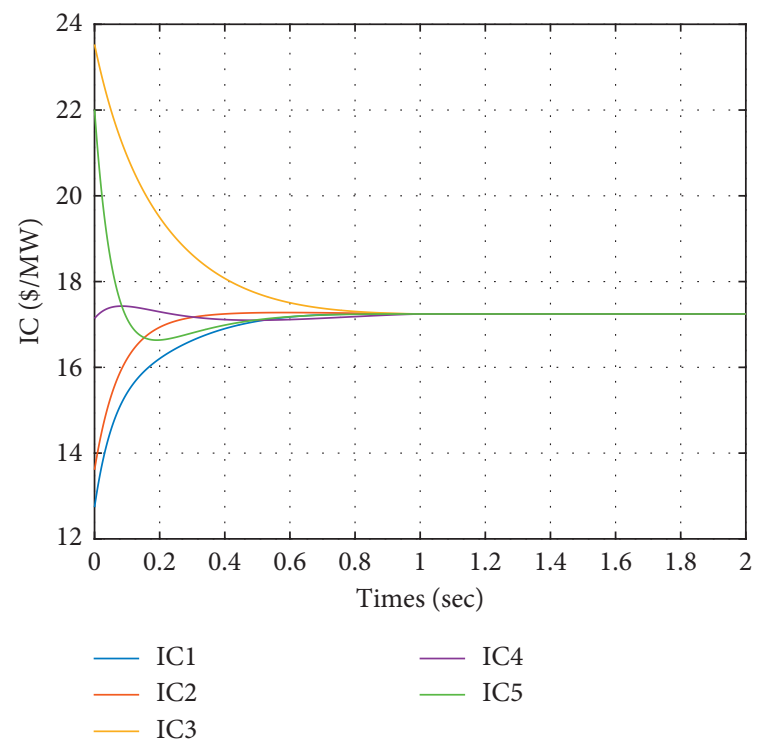

FIGURE 11: The incremental cost of generator $\lambda_{i}$ with the valve point effect.

(33) and (34), $\hat{x}_{i}^{*}=1.74$ in Figure 7 and $\hat{y}_{i}^{*}=4.35$ in Figure 8 reach consensus in 5 s. From (37), we can get the final incremental cost $\lambda^{*}=17.43$. It can be seen from Figure 9 that the total generator demand is $437.67 \mathrm{MW}$, which satisfies the balance condition of power demand. From Figure 10, we found that each $P_{i}$ will reach a value in prescribed finite time, that is, $P_{1}^{* *}=82.35 \mathrm{MW}, P_{2}^{* *}=108.48 \mathrm{MW}$, $P_{3}^{* *}=73.81 \mathrm{MW}, P_{4}^{* *}=97.63 \mathrm{MW}$, and $P_{5}^{* *}=75.40 \mathrm{MW}$.

4.3. Prescribed Finite Time Consensus with the Valve Point Effect. According to (23), $d_{1}=0.45, d_{2}=0.6, d_{3}=0.32$, $d_{4}=0.26, d_{5}=0.33, e_{1}=0.041, e_{2}=0.036, e_{3}=0.028$,

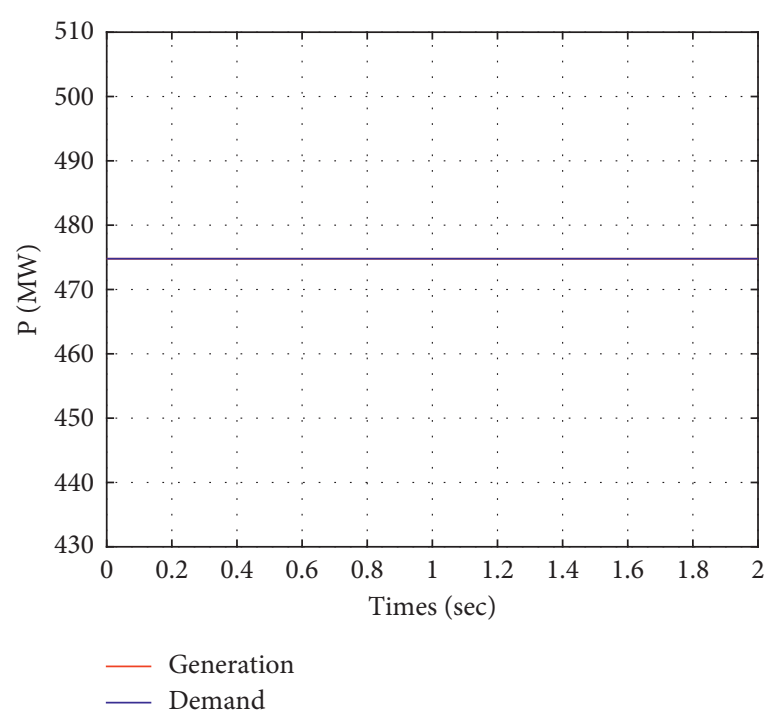

Figure 12: Total power demand of generator with the valve point effect.

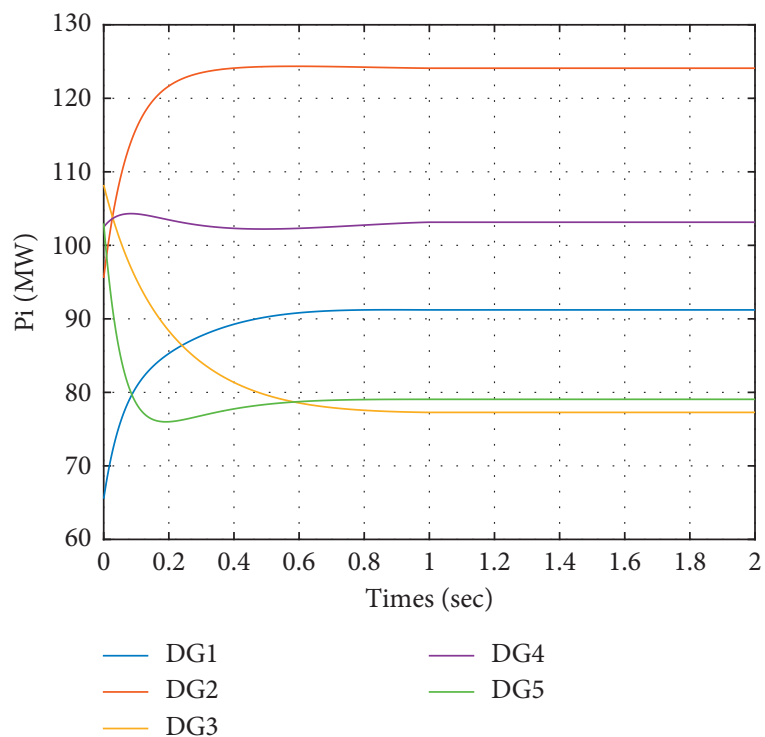

FIgURE 13: The output power of generator $P_{i}$ with the valve point effect.

$e_{4}=0.052$, and $e_{5}=0.031$ are selected in this paper. In line with formula (28), we can set the convergence time as 1s. The incremental cost of the generator with the valve point effect is $\lambda^{*}=17.24$, as shown in Figure 11. The total power requirement is $474.79 \mathrm{MW}$, as in Figure 12, which meets the balance of power demand and supply. From Figure 13, it is found that all $P_{i}$ can reach consensus in prescribed finite time, where the steady states are $P_{1}=$ 91.22 MW, $P_{2}=124.10 \mathrm{MW}, P_{3}=77.28 \mathrm{MW}$, $P_{4}=103.14 \mathrm{MW}$, and $P_{5}=79.05 \mathrm{MW}$.

\section{Conclusions}

This paper has solved the distributed prescribed finite time optimization ED problem with and without the valve point effect. The relationship between the consensus and 
optimization problem of ED has been derived, and we can easily transform the optimization problem into a consensus problem. For both an undirected network and balance directed network, the prescribed finite time consensus schemes for ED of smart grids have been investigated. We found that if there exist constraints of generation units, the prescribed finite time optimization ED problem can also be solved.

\section{Data Availability}

The authors pledge to provide all the codes and the data underlying the findings of the study.

\section{Conflicts of Interest}

The authors declare that there are no conflicts of interest regarding the publication of this paper.

\section{Acknowledgments}

The authors would like to thank the National Natural Science Foundation of China (grant nos. 61603196 and 61873326) for supporting this research work.

\section{References}

[1] A. Bakirtzis, V. Petridis, and S. Kazarlis, "Genetic algorithm solution to the economic dispatch problem," IEEE Proceedings-Generation, Transmission and Distribution, vol. 141, pp. 377-382, 2002.

[2] P. Chen and H. Chang, "Large-scale economic dispatch by genetic algorithm," IEEE Transactions on Power Systems, vol. 10, no. 4, pp. 1919-1926, 1995.

[3] Z.-L. Gaing, "Particle swarm optimization to solving the economic dispatch considering the generator constraints," IEEE Transactions on Power Systems, vol. 18, no. 3, pp. 1187-1195, 2003.

[4] J. Park, Y. Jeong, J. Shin, and K. Lee, “An improved particle swarm optimization for nonconvex economic dispatch problems," IEEE Transactions on Power Systems, vol. 25, no. 1, pp. 156-166, 2010.

[5] J.-B. Park, K.-S. Lee, J.-R. Shin, and K. Y. Lee, "A particle swarm optimization for economic dispatch with nonsmooth cost functions," IEEE Transactions on Power Systems, vol. 20, no. 1, pp. 34-42, 2005.

[6] F. Yao, Z. Y. Dong, K. Meng, Z. Xu, H. H.-C. Iu, and K. P. Wong, "Quantum-inspired particle swarm optimization for power system operations considering wind power uncertainty and carbon tax in Australia," IEEE Transactions on Industrial Informatics, vol. 8, no. 4, pp. 880-888, 2012.

[7] X. Xu, Z. Hu, Q. Su, and Z. Xiong, "Multiobjective collective decision optimization algorithm for economic emission dispatch problem," Complexity, vol. 2018, Article ID 1027193, 20 pages, 2018.

[8] Z. Zhang and M. Chow, "Convergence analysis of the incremental cost consensus algorithm under different communication network topologies in a smart grid," IEEE Transactions on Power Systems, vol. 27, no. 4, pp. 761-1768, 2012.

[9] Z. Zhang and M. Chow, "Decentralizing the economic dispatch problem using a two-level incremental cost consensus algorithm in a smart grid environment," in Procedings of the
IEEE 2011 North American Power Symposium, Boston, MA, USA, August 2011.

[10] Z. Zhang and M. Chow, "The influence of time delays on decentralized economic dispatch by using incremental cost consensus algorithm," in Control and Optimization Methodsfor Electric Smart Grids, Power Electronics and Power Systems, A. Chakrabortty and M. D. Ilic, Eds., pp. 313-326, Springer Science Business Media, Berlin, Germany, 2012.

[11] W. Yu, C. Li, X. Yu, G. Wen, and J. Lü, "Distributed consensus strategy for economic power dispatch in a smart grid," in Proceedings of the 10th Asian Control Conference, Sabah, Malaysia, June 2015.

[12] G. Wen, X. Yu, Z.-W. Liu, and W. Yu, "Adaptive consensusbased robust strategy for economic dispatch of smart grids subject to communication uncertainties," IEEE Transactions on Industrial Informatics, vol. 14, no. 6, pp. 2484-2496, 2018.

[13] G. Chen and Z. Zhao, "Delay effects on consensus-based distributed economic dispatch algorithm in microgrid," IEEE Transactions on Power Systems, vol. 33, no. 1, pp. 602-612, 2018.

[14] Y. Fan, G. Hu, and M. Egerstedt, "Distributed reactive power sharing control for microgrids with event-triggered communication," IEEE Transactions on Control Systems Technology, vol. 25, no. 1, pp. 118-128, 2017.

[15] G. Chen, Z. Li, and Z. Zhao, "Event-triggered optimal active power control in islanded microgrid with variable demand and time-varying communication topology," IEEE Transactions on Smart Grid, vol. 10, no. 4, pp. 4015-4025, 2019.

[16] C. Li, X. Yu, W. Yu, T. Huang, and Z.-W. Liu, "Distributed event-triggered scheme for economic dispatch in smart grids," IEEE Transactions on Industrial Informatics, vol. 12, no. 5, pp. 1775-1785, 2016.

[17] X. He, D. W. C. Ho, T. Huang, J. Yu, H. Abu-Rub, and C. Li, "Second-order continuous-time algorithms for economic power dispatch in smart grids," IEEE Transactions on Systems, Man, and Cybernetics: Systems, vol. 48, no. 9, pp. 1482-1492, 2018.

[18] C. Huang, X. Zhang, H. Lam, and S. Tsai, "Synchronization analysis for nonlinear complex networks with reaction-diffusion terms using fuzzy-model-based approach," IEEE Transactions on Fuzzy Systems, vol. 2020, Article ID 2974143, 13 pages, 2020.

[19] C. Huang, J. Lu, G. Zhai, J. Cao, G. Lu, and M. Perc, "Stability and stabilization in probability of probabilistic boolean networks," IEEE Transactions on Neural Networks and Learning Systems, vol. 2020, Article ID 2978345, 11 pages, 2020.

[20] C. Huang, J. Lu, D. W. C. Ho, G. Zhai, and J. Cao, "Stabilization of probabilistic boolean networks via pinning control strategy," Information Sciences, vol. 510, pp. 205-217, 2020.

[21] G. Chen, Z. Zhao, and Z. Li, "Distributed finite-step iterative algorithm for economic dispatch of generation," IEEE Transactions on Industrial Informatics, vol. 14, no. 12, pp. 5221-5232, 2018.

[22] G. Chen and Z. Li, "Consensus based distributed finite-time economic dispatch in smart grid with jointly connected topology," in Proceedings of the 29th Chinese Control and Decision Conference, Chongqing, China, May 2017.

[23] G. Chen, J. Ren, and E. Feng, "Distributed finite-time economic dispatch of a network of energy resources," IEEE Transactions on Smart Grid, vol. 8, no. 2, pp. 822-832, 2017.

[24] Y. Song, Y. Wang, J. Holloway, and M. Krstic, "Time-varying feedback for regulation of normal-form nonlinear systems in 
prescribed finite time," Automatica, vol. 83, pp. 243-251, 2017.

[25] H. Xiang, G. Chen, and Z. Zhao, "A distributed algorithm for economic dispatch in prescribed time," IFAC-PapersOnLine, vol. 52, no. 24, pp. 184-189, 2019.

[26] X. Li, D. W. C. Ho, and J. Cao, "Finite-time stability and settling-time estimation of nonlinear impulsive systems," Automatica, vol. 99, pp. 361-368, 2019.

[27] G. Mei, X. Wu, D. Ning, and J.-A. Lu, "Finite-time stabilization of complex dynamical networks via optimal control," Complexity, vol. 21, no. S1, pp. 417-425, 2016.

[28] H. Yang, P. Yang, and C. Huang, "Evolutionary programming based economic dispatch for units with nonsmooth fuel cost functions," IEEE Transactions on Power Systems, vol. 11, no. 1, pp. 112-118, 1996.

[29] N. Sinha, R. Chakrabarti, and P. Chattopadhyay, "Evolutionary programming techniques for economic load dispatch," IEEE Transactions on Evolutionary Computation, vol. 7, no. 1, pp. 822-832, 2003.

[30] D. Walters and G. Sheble, "Genetic algorithm solution of economic dispatch with valve point loading," IEEE Transactions on Power Systems, vol. 8, no. 3, pp. 184-189, 1993.

[31] S. Ling, H. Lam, F. Leung, and Y. LEE, "Improved genetic algorithm for economic load dispatch with valve-point loadings," in Proceedings of the 29th Annual Conference of the IEEE Industrial Electronics Society, Roanoke, VA, USA, November 2003.

[32] D. He, F. Wang, and Z. Mao, "A hybrid genetic algorithm approach based on differential evolution for economic dispatch with valve-point effect," International Journal of Electrical Power \& Energy Systems, vol. 30, no. 1, pp. 31-38, 2008.

[33] C.-L. Chiang, "Improved genetic algorithm for power economic dispatch of units with valve-point effects and multiple fuels," IEEE Transactions on Power Systems, vol. 20, no. 4, pp. 1690-1699, 2005.

[34] T. Victorie and A. Jeyakumar, "Hybrid PSO-SQP for economic dispatch with valve-point effect," Electric Power Systems Research, vol. 71, no. 1, pp. 51-59, 2004.

[35] X. Chen, B. Xu, and W. Du, "An improved particle swarm optimization with biogeography-based learning strategy for economic dispatch problems," Complexity, vol. 2018, Article ID 7289674, 15 pages, 2018.

[36] S. Hemamalini and S. P. Simon, "Maclaurin series-based Lagrangian method for economic dispatch with valve-point effect," IET Generation, Transmission \& Distribution, vol. 3, no. 9, pp. 859-871, 2009.

[37] M. Abouheaf, W. Lee, and F. Lewis, "Dynamic formulation and approximation methods to solve economic dispatch problems," IET Generation Transmission and Distribution, vol. 7, no. 8, pp. 866-873, 2003.

[38] F. Li, J. Qin, and Y. Kang, "Multi-agent system based distributed pattern search algorithm for non-convex economic load dispatch in smart grid," IEEE Transactions on Power Systems, vol. 34, no. 3, pp. 2093-2102, 2019.

[39] T. Nguyen, N. Quynh, and L. Dai, "Improved Firefly Algorithm: a novel method for optimal operation of thermal generating units," Complexity, vol. 2018, Article ID 7267593, 23 pages, 2018.

[40] R. Morsali, M. Mohammadi, I. Maleksaeedi, and N. Ghadimi, "A new multiobjective procedure for solving nonconvex environmental/economic power dispatch," Complexity, vol. 20, no. 2, pp. 47-62, 2015.

[41] L. Yang, D. He, and B. Li, "A selection hyper-heuristic algorithm for multiobjective dynamic economic and environmental load dispatch," Complexity, vol. 2020, Article ID 4939268, 18 pages, 2020.

[42] Y. Elsheakh, S. Zou, Z. Ma, and B. Zhang, "Decentralised gradient projection method for economic dispatch problem with valva point effect," IET Generation Transmission \& Distribution, vol. 34, no. 3, pp. 3844-3851, 2018. 